\title{
COMPARATIVE ANALYSIS OF COLON CANCER VS. RECTAL CANCER IN SENTINEL LYMPH NODE MAPPING
}

\author{
Ignatov V., K. Ivanov, N. Kolev, A. Tonev \\ Department of General and Operative Surgery, University Hospital "St. Marina"
}

Reviewed by: Prof. R. Madjov

\begin{abstract}
INTRODUCTION: The lymphatic status is acknowledged as the most important prognostic factor in patients with colorectal cancer. In our clinic, the intraoperative sentinel lymph node mapping with Patent Blue $V$ is a routine method of choice for better staging of lymph node status and achieving an adequate extent of surgical procedure in patients with colorectal cancer. AIM: To compare the results from application of methods of intraoperative sentinel lymph node mapping in patients with colon cancer vs patients with rectal cancer. RESULTS: There were 136 consecutive patients (65 with colon and 71 with rectum). The sentinel lymph nodes were identified in 100 percent of colon and rectal patients. Skip metastases were found in $\mathbf{3 . 0}$ percent of colon vs. 2.81 percent of rectal patients. Occult micrometastases were found in 9 percent of colon vs. 7.0 percent of rectal patients. No other parameters were statistically different between colon and rectum. The study was undergone in $\mathbf{1 3 6}$ patients with diagnosis of colorectal cancer and sentinel lymph node mapping. An analysis and comparison is done and the results of the two methods were compared. Our data show that the sensitivity is comparable and is respectively $100 \%$ and $95 \%$, respectively for the colon and rectum, the methods are reliable enough. CONCLUSION: The surgical approach and the extent of the lymph dissection have to be conformable to the status of lymph node basin, staged with the help of objective intraoperative diagnostic tool, such as intraoperative sentinel lymph node mapping. This leads to increasing level of curative surgical treatment in cases of colorectal cancer. Despite higher success rates in sentinel lymph node identification for colon patients, sentinel lymph node mapping was highly successful (91.5 percent) in rectal patients. Nodal upstaging, skip metastases, and occult metastases were analysed.
\end{abstract}

Key words: Sentinel lymph node mapping, colon and rectum, caner

In the diagnostics, staging and treatment of the patients with colorectal cancer are included specialists from many different areas of medicine: surgeons, oncologists, specialists in imaging diagnostics, radiotherapists, pathomorphologists, genetics and etc. The surgeon is the main force in those processes. The method of choice is based upon complex of criteria and data about the stage of differentiation, a presence of extra cellular or intracellular mucous secreting, the stage of tumor cell invasion in the lymphatics, arterioles, venulas and in perineural space, the angiogenesis of the tumor, the mitotic index, the grade of lymphocytic infiltration in the tumor and the count of T-lymphocytes in the peripheral blood. The molecular and genetic prognostic factors from the primary tumor and metastatic origin, as well as morphological exploration and the ultrastaging of oncological disease are main fields of investigation.

The surgical treatment is the base of complex medical treatment, which aims to cure definitely the patients with colorectal cancer. Good results from the curative process

Address for correspondence:

Kr.Ivanov, Varna, 1 Hr. Smirnenski str,

University Hospital "St. Marina"

e-mail: teraton@abv.bg, kivanov@gisurgery.bg are obtained by modeling an individual protocol to every single patient which conformable to the present medical consensuses.

The quality of a surgical treatment is defined as well as the choice of appropriate operative approach, but also from the tumor characteristics, the vessels that uptake the lymph flow, and the modalities for preoperative and intraoperative staging.

Undergoing an operation in adequate extent is a premise for precise morphological exploration of the oncological disease and defines the approach in the postoperative treatment. The curative procedures gain the best effect when the patient is diagnosed in I-st or II-nd clinical stage of the oncological disease, before the presence of tumor metastasis.

The lymph node status is the most important prognostic factor for patients with colorectal cancer. The exact preoperative staging of the lymph node status is unsettled problem because of the sensitive methods for preoperative diagnosis - PET, CT, MRI and radioimmunoguided detection cannot be used as routine methods in every patient. The clinical examination and intraoperative exploration are not so sensitive and specific methods and could only to led the surgeon in certain direction. There aren't established special intraoperative diagnostic methods for the lymphatic status, in accordance with the surgeon can change his operative behavior for obtaining à radical treatment. The standard histopmorphological intraoperative 
examination is low-sensitive and can not to define the presence or absence of micrometastasis in lymph nodes. One of the present problems is the inability to exact determination of the lymph node status during the standard morphological examination of resected specimen. The morphologists can not obtain enough lymph nodes and the ultrastaging techniques are not introduced in the routine practice for the lymph nodes with highest metastatic potential - the sentinel lymph nodes. The consequences of non-precised examination of lymph node basin lead to developing of recurrence in $1 / 3$ of the patients underwent curative resections for non - metastatic colorectal cancer.

The first report of SLNM in colorectal cancer was a feasibility study presented in 1997 at the Society of Surgical Oncology's $50^{\text {th }}$ Annual Cancer Symposium in Chicago (1). This series of 10 patients was followed by a series of 56 patients presented at the 17th International Cancer Congress in Brazil in 1998 (2). This report confirmed the high (98\%) feasibility of the technique and demonstrated its high (95\%) degree of accuracy for predicting the status of the nodal basin. In 1999, Joosten et al. (3) in the Netherlands published a report of SLNM in 50 patients with colorectal cancer. On the basis of a feasibility rate of $70 \%$ and a false-negative rate of $60 \%$, they concluded that SLNM was not a reliable method of staging colorectal cancer. However, their injection method and especially the timing of pathological examination differed from those described by us. Their results underscore the importance of adhering to strict technical details of the procedure and following a specific protocol for handling and processing of the specimen by the pathologist. A subsequent publication in the Annals of Surgical Oncology (4) outlined the technical details associated with excellent feasibility (99\%) and accuracy (96\%) rates as reported in our original studies.

In December 2000, a review by Saha et al. (5) Include coauthor Wong's technique of ex vivo lymphatic mapping in colorectal cancer. Kitagawa's group 6 focused on the use of SLNM in esophageal, gastric, and colorectal cancers and for the first time described the use of radiolabeled tracers and a hand-held gamma probe for successful lymphatic mapping in colorectal cancer. Thorn (7) in 2000 and Merrie et al. (8) in 2001 combined blue dye and radioactive tracer for mapping, as previously described in breast and melanoma. The success rates of dual-agent mapping were $100 \%$ (7) and $88 \%$ (8). In 2001, Wood et al. (9) reported a series of 75 patients who underwent lymphatic mapping by in vivo, ex vivo and laparoscopic techniques (9). The overall success rate of mapping was $96 \%$, and the rate of occult micrometastasis was $17 \%$.

The first large series of ex vivo SLNM in colon and rectal cancers was published by Wong et al. in 2001 (10). Laparoscopic SLNM has been attempted at a few centers for colorectal and gastric cancers, with high success rates $(9,11)$ comparable to those associated with mapping performed during open laparotomy. Also in 2001, Bilchik et al. (12) first reported molecular profiling of colon cancer with use of the RT-PCR technique for multiple markers in 40 patients. This study showed a high correlation between the expression of $\mathrm{p} 53$, beta-HCG, c-Met, and uMAGE in a primary tumor and the presence of micrometastases in regional lymph nodes.

Aim: To compare the results from application of methods of intraoperative sentinel lymph node mapping in patients with colon cancer vs. patients with rectal cancer.

\section{MATERIAL}

We made a prospective study for a period of $2004-2006$, in 242 patients, operated for colorectal cancer. The investigation was conducted in the clinic of general and operative surgery in the University Hospital "St. Marina", Varna. In 136 of the cases we applied the method of intraoperative sentinel lymph node mapping.

\section{METHODS}

We applied the next modes of lymphatic mapping:

1. Intraoperative sentinel lymph node mapping in 71 patients with rectal cancer.

2. Intraoperative sentinel lymph node mapping in 65 patients with colon cancer.

\section{Statistics:}

In the statistical processing of our data we used the following methods:

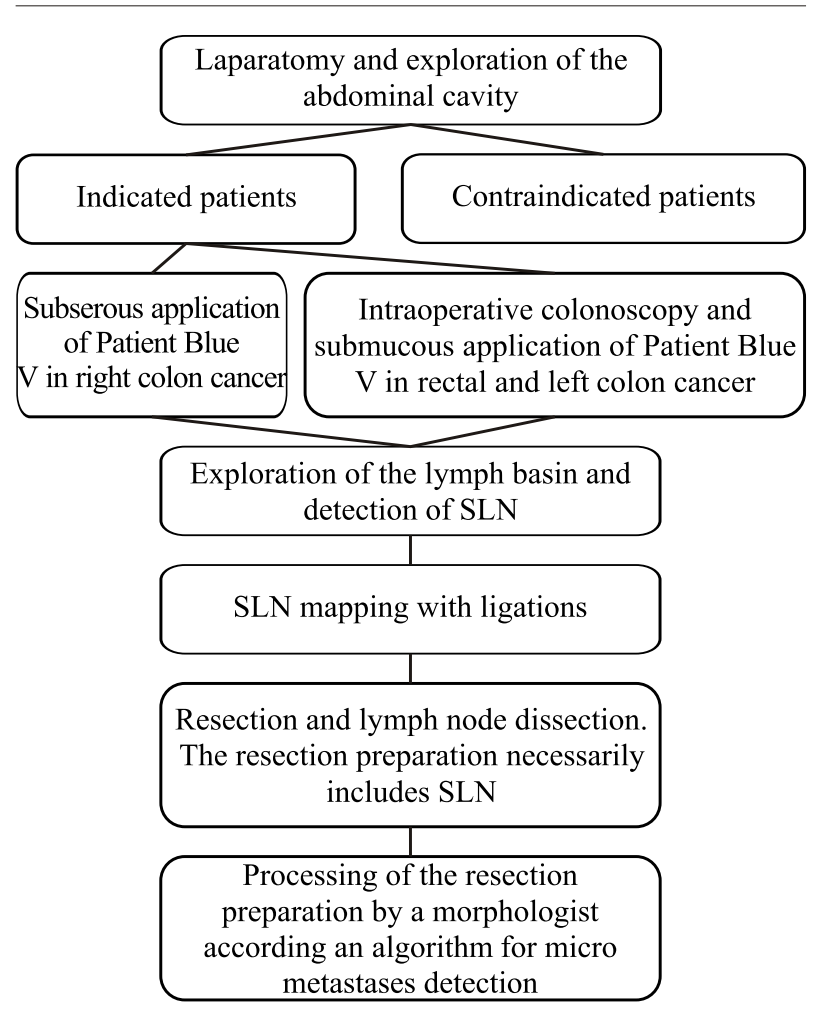

Fig. 1 Algorithm for sentinel mapping in colorectal cancer

1. Estimating the indexes for the relative share.

2. Estimating of the average values. 


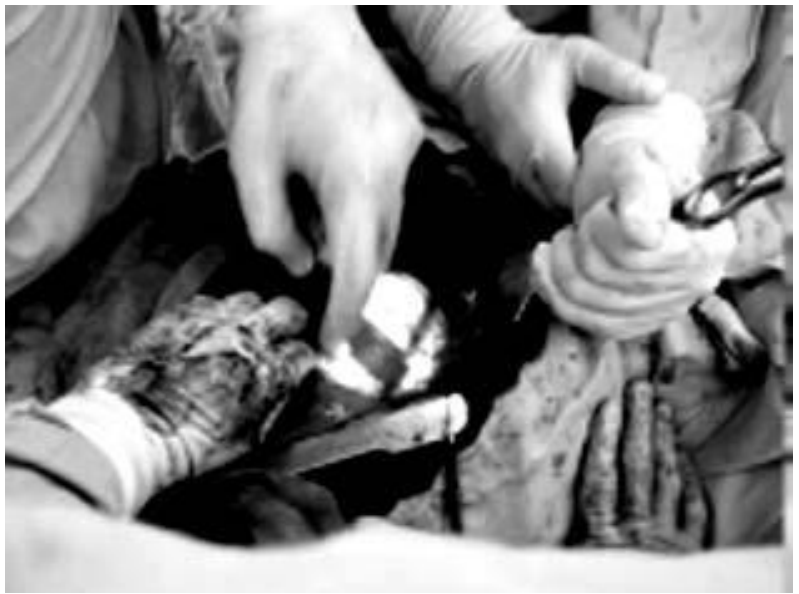

Fig. 2. Intraoperative colonoscopy

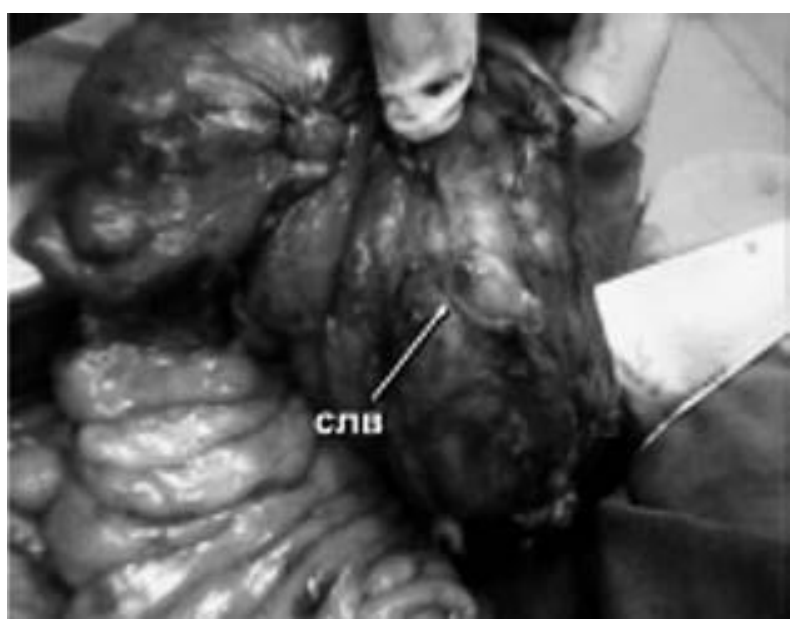

Fig. 4. Laparoscopic view of sentinel lymph node of mesorectum

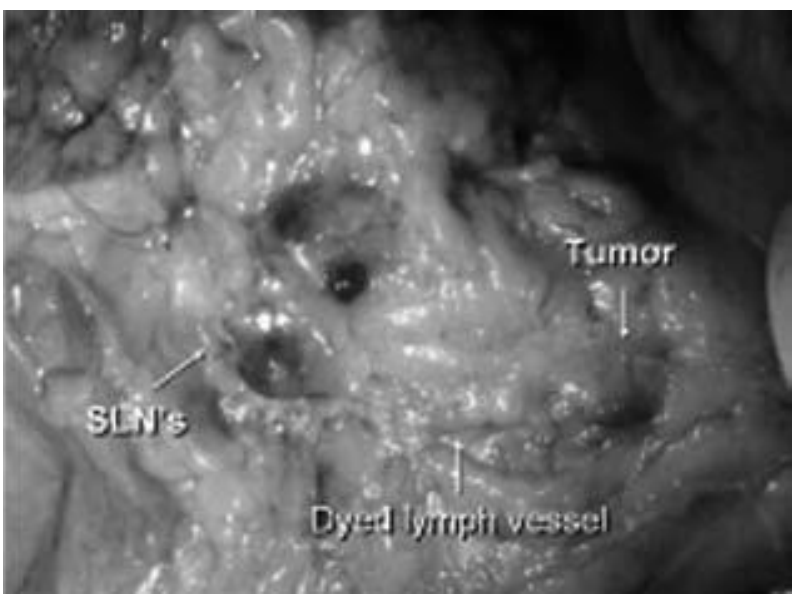

Fig. 6. Tumor of the colon, peritumoral injecting of Patent Blue V dye. Gained direct visualization of the lymphatic, draining the primary tumor to tree sentinel lymph nodes

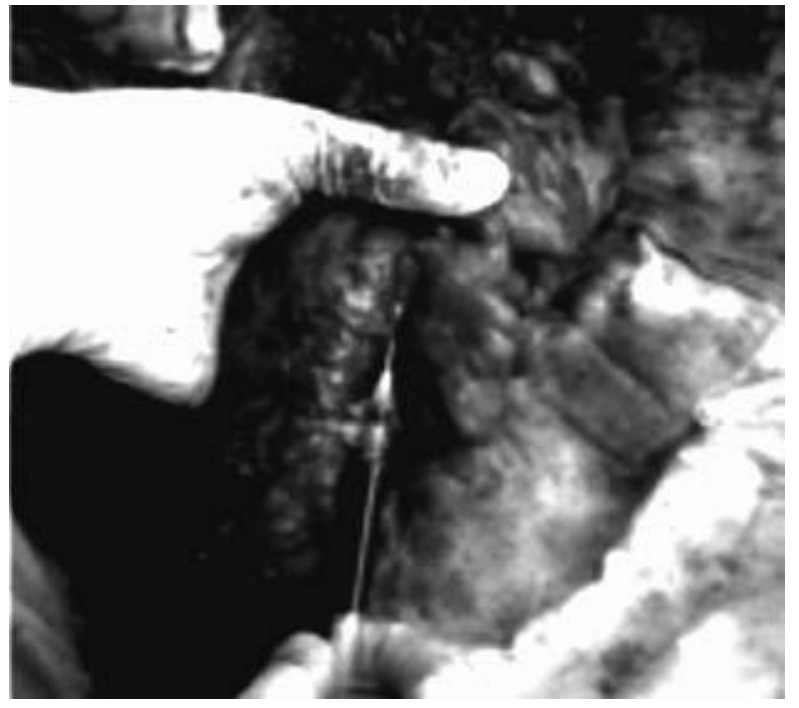

Fig. 3. Subserosal application of Patent Blue dye

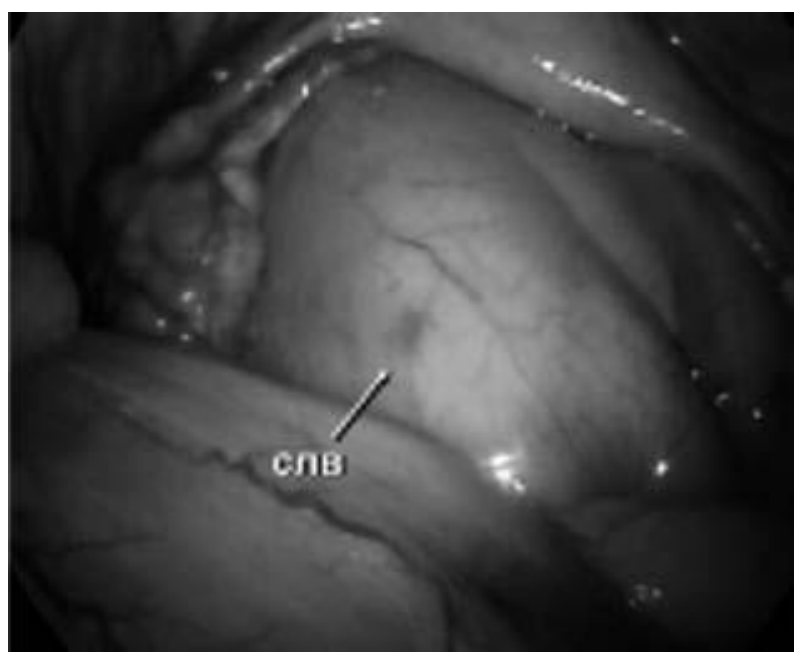

Fig. 5. Laparoscopic view of sentinel lymph node of mesorectum

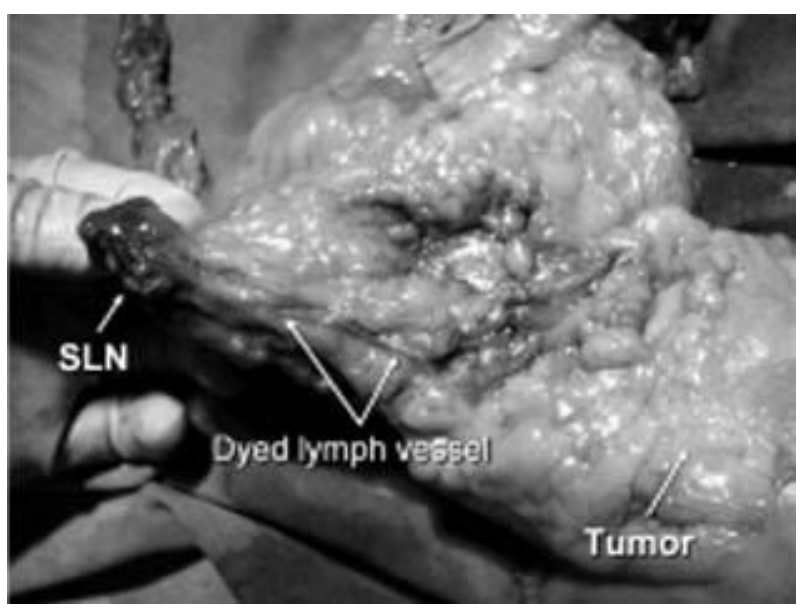

Fig. 7. Dyed with Patent Blue Vis demonstrated on the figure conetcting the tumor with a sentinel lymph node from third level 
Comparative analysis of colon cancer vs. rectal cancer ...

3. Tables

4. Charts

We developed and suggest the following algorhytm for intraoperative sentinel lymph node mapping in patients of colon and rectal cancer.

\section{RESULTS}

We present the distribution of the patients in localization, sex and age in the following:

We found metastases and micrometastases in 57\% (112 of 196) of the SLNs, compared with 9\% (198 of 2208) in non-SLNs.

In case of absence of metastases in SLNs, the possibility for presence of non-SLNs is only $0.6 \%$ (4 of 658).

From the group of 136 patients, 65 (48\%) of them were

\begin{tabular}{||c|c|c|c||}
\hline \hline $\begin{array}{c}\text { LOCALIZATION } \\
\text { GROUPS OF } \\
\text { PATIENTS }\end{array}$ & $\begin{array}{c}\text { COLON } \\
\text { CANCER }\end{array}$ & $\begin{array}{c}\text { RECTAL } \\
\text { CANCER }\end{array}$ & TOTAL \\
\hline Patients (n) & 65 & 71 & 136 \\
\hline Male (n) & 30 & 33 & 63 \\
\hline Female (n) & 35 & 38 & 73 \\
\hline Average age (years) & 63 & 66 & 65 \\
\hline \hline
\end{tabular}

Fig 8. Distribution of the patients

\begin{tabular}{|l|c|c||}
\hline $\begin{array}{l}\text { LOCALIZATION } \\
\text { LYMPH NODES }\end{array}$ & COLON CANCER & $\begin{array}{l}\text { RECTAL } \\
\text { CANCER }\end{array}$ \\
\hline Lymph nodes $(\mathrm{n})$ & 15,2 & 13,6 \\
\hline
\end{tabular}

Fig. 9. Average numbers of lymph nodes and sentinel lymph nodes in the resected specimen

\begin{tabular}{||l|c|c||}
\hline \hline PATIENTS (N) & $\mathbf{N}$ & $\mathbf{\%}$ \\
\hline 1 & 54 & 40 \\
\hline 2 & 53 & 39 \\
\hline 3 & 26 & 19 \\
\hline 4 & 3 & 2 \\
\hline
\end{tabular}

Fig. 10. Distribution of the patients in dependence of founded sentinel lymph nodes

with Ò1,2 - stage of the primary tumor; 57 (42\%) of them were with Ò - stage of the primary tumor; $14(10 \%)$ of them were with OA - stage of the primary tumor. It is underlined that from the Ò1,2 group of 65 patients, 20 (31\%) of them were with presence of LN's metastases and $10(15 \%)$ of them were with micrometases.

From the Ò3 group of 57 patients, 38 (66\%) of them were with presence of LN's metastases and $4(7 \%)$ of them were with micrometases. From the Oे group of 14 patients, 14

\begin{tabular}{||l|c|c|c|c|c|c||}
\hline \multirow{2}{*}{$\begin{array}{l}\text { Localization } \\
\text { Cases }\end{array}$} & \multicolumn{2}{|c|}{ Colon cancer } & \multicolumn{2}{|c||}{ Rectal cancer } & \multicolumn{2}{|c||}{ Total } \\
\cline { 2 - 7 } & $\mathbf{n}$ & $\mathbf{\%}$ & $\mathbf{n}$ & $\mathbf{\%}$ & $\mathbf{n}$ & $\mathbf{\%}$ \\
\hline Patients & 65 & 48 & 71 & 52 & 136 & 100 \\
\hline $\begin{array}{l}\text { Successful } \\
\text { SLNM }\end{array}$ & 65 & 100 & 71 & 100 & 136 & 100 \\
\hline $\begin{array}{l}\text { Presence of LN } \\
\text { metastases }\end{array}$ & 34 & 52 & 38 & 54 & 72 & 53 \\
\hline \begin{tabular}{l} 
False-negative \\
\hline $\begin{array}{l}\text { Metastases only } \\
\text { in SLNs }\end{array}$
\end{tabular} & - & - & 3 & 4 & 3 & 2 \\
\hline $\begin{array}{l}\text { Founded } \\
\text { micrometastases }\end{array}$ & 6 & 9 & 8 & 11 & 14 & 10 \\
\hline
\end{tabular}

Fig. 11. Summarized results from the application of intraoperative sentinel lymph node mapping in cases of colorectal cancer

\begin{tabular}{||l|c|c|c|c|c|c||}
\hline \hline \multirow{2}{*}{$\begin{array}{l}\text { Ostage } \\
\text { Metastases in LN }\end{array}$} & \multicolumn{2}{|c|}{ T1,2 } & \multicolumn{2}{c|}{ T3 } & \multicolumn{2}{c||}{ T4 } \\
\cline { 2 - 8 } & $\mathbf{n}$ & $\mathbf{n}$ & $\mathbf{n}$ & $\mathbf{\%}$ & $\mathbf{n}$ & $\mathbf{\%}$ \\
\hline Patients & 65 & 48 & 57 & 42 & 14 & 10 \\
\hline $\begin{array}{l}\text { Presence of LN's } \\
\text { metastases }\end{array}$ & 20 & 31 & 38 & 66 & 14 & 100 \\
\hline Micrometastases & 10 & 15 & 4 & 7 & - & - \\
\hline \hline
\end{tabular}

Fig. 12. Ratio between T-stage of the primary tumor and the presence of lymph node metastases after application of SLNM

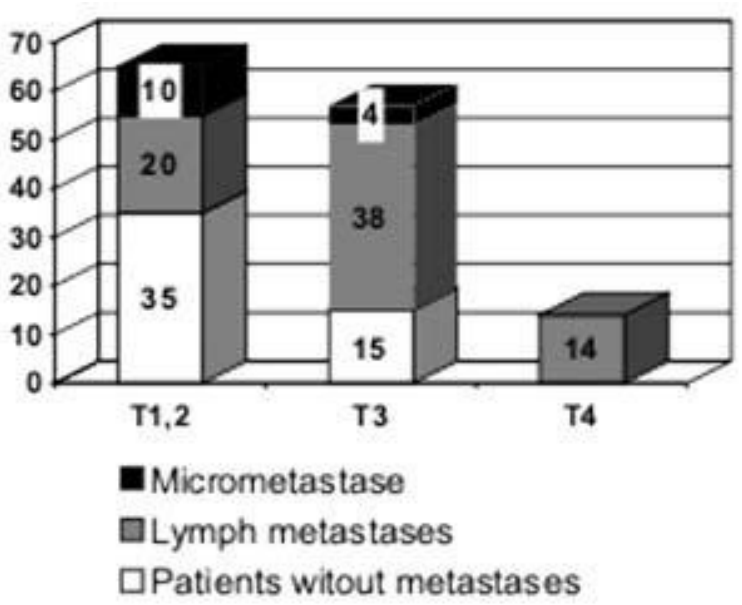

Fig. 13. Ratio between T-stage of the primary tumor and the presence of lymph node metastases after application of SLNM

$(100 \%)$ of them were with presence of LN's metastases and $0(0 \%)$ of them were with micrometases. It is demonstrated that the micrometastases were founded more frequently in cases of lower T-stage of the primary tumor. 


\section{CONCLUSION}

1. The method of intraoperative sentinel lymph node mapping with Patent Blue V is an accurate and objective diagnostic method for evaluating of lymph basin status in patients with colorectal cancer. The method is an objective intraoperative criterion for determination of surgical decision making. .

2. The method of intraoperative sentinel lymph node mapping is feasible and has a high success in patients with colorectal cancer. The method is high successful and sensitive, respectively $100 \%$ and $98 \%$.

\section{REFERENCES}

1. Saha S, Ganatra BK, Gauthier J, et al. Localization of sentinel lymph node in colon cancer. A feasibility study. SSO $50^{\text {th }}$ Annual Cancer Symposium 1997; 80: 54.

2. Saha S, Espinosa M, Wiese D, et al. Accurate staging of colorectal cancer by sentinel lymph node mapping. A prospective study. Proceedings of the $17^{\text {th }}$ International Cancer Congress 1998;2:1005-11.

3. Joosten JJ, Strobbe LJ, Wauters CA, et al. Intraoperative lymphatic mapping and the sentinel node concept in colorectal carcinoma. Br J Surg 1999; 86: 482-6.

4. Saha S, Wiese D, Badin J, et al. Technical details of sentinel lymph node mapping in colorectal cancer and its impact on staging. Ann Surg Oncol 2000; 7: 120-4.

5. Saha S, Nora D, Wong JH, et al. Sentinel lymph node mapping in colorectal cancer-a review. Surg Clin Of N Am 2000; 80: 1811-9.

6. Kitagawa Y, Fugii H, Mukai M, et al. The role of the sentinel lymph node in gastrointestinal cancer. Surg Clin North Am 2000; 80: 1799-809.

7. Thorn M. Lymphatic mapping and sentinel node biopsy: is the method applicable to patients with colorectal and gastric cancer? Eur J Surg 2000; 166: 755-8.

8. Merrie AE, van Rij AM, Phillips LV, et al. Diagnostic use of the sentinel node in colon cancer. Dis Colon Rectum 2001; 44: 410-7.

9. Wood TF, Saha S, Morton DL, et al. Validation of lymphatic mapping in colorectal cancer: in vivo, ex vivo, and laparoscopic techniques. Ann Surg Oncol 2001; 8: 150-7.

10. Wong JH, Steineman S, Calderia C, et al. Ex vivo sentinel node mapping in carcinoma of the colon and rectum. Ann Surg 2001; 233: 515-21

11. Kitagawa Y, Ohgami M, Fujii H, et al. Laparoscopic detection of sentinel lymph nodes in gastrointestinal cancer: a novel and minimally invasive approach. Ann Surg Oncol 2001; 8: 86-9S.

12. Bilchik AJ, Saha S, Wiese D, et al. Molecular staging of early colon cancer on the basis of sentinel node analysis: a multicenter phase II trial. J Clin Oncol 2001; 19: 1128-36. 